\title{
Immunohistochemical and Quantitative Analysis of Cellular and Extra- cellular Components of Aortic Atherosclerosis in WHHL Rabbits
}

\author{
Takashi Ito', Toyohiro Tsukada², Makiko Ueda ${ }^{3}$, Hideki Wanibuchi', \\ and Masashi Shiomi' \\ ${ }^{1}$ Institute for Experimental Animals, Kobe University School of Medicine, Kobe, Japan. ${ }^{2}$ Second Department of \\ Internal Medicine, Sanraku Hospital, Tokyo, Japan. ${ }^{3}$ First Department of Pathology, Osaka City University Medical \\ School, Osaka, Japan.
}

\begin{abstract}
To investigate changes in the major components of atherosclerotic lesions during the progression of this disease, we measured the lesional areas of macrophages, smooth muscle cells, collagen fibers, and extracellular lipid deposits in the aortas of WHHL rabbits. Aortic segments with lesions of various stages were stained for histological and immunohistochemical examination, and the area of each lesional component was measured by a color image analyzer. In the early fatty streaks observed in 3-month-old rabbits, macrophages were predominant in the intima and were also observed in the inner layer of the media. In the transitional lesions (fibro-fatty streaks) found in rabbits at 11 to 15 months of age, an increase in the lesional area of macrophages was prominent compared to other lesional components. Thus, macrophages may play an important role in the progression of aortic atherosclerosis at this stage. In advanced complicated lesions observed in rabbits at 20 to 24 months of age, the area of macrophages and smooth muscle cells did not increase, whereas the area of collagen fibers and extracellular lipid deposits increased. Therefore, both the disruption of foam cells and fibrosis may play an important role in the progression of atherosclerosis at this stage. J Atheroscler Thromb, $1994 ; 1$ : 45-52.
\end{abstract}

Key words : Macrophages, Smooth muscle cells, Aortic atherosclerosis, WHHL rabbit

The response-to-injury hypothesis of atherogenesis was proposed by Ross and Glomset in 1976 (1), and was revised by Ross in $1986(2)$. The revised hypothesis proposed that both the type of lesion and its components changed as atherosclerosis progressed. It is well known that atherosclerotic lesions consist of macrophages, smooth muscle cells, and lymphocytes as well as an extracellular matrix composed of collagen, elastin, fibrin, calcium, and lipid deposits. These components are seen in WHHL rabbits (3-7), a genetic animal model of patients with familial hypercholesterolemia $(8,9)$, and various cholesterol-fed animal models, including nonhuman primates $(10-12)$, swine $(13,14)$, rabbits $(15-18)$, rats (19) and pigeons

Address for correspondence: Masashi Shiomi, PhD., Institute for Experimental Animals, Kobe University School of Medicine, Kusunoki-Cho, Chuo-ku, Kobe 650, Japan.

Received December 22, 1992.

Accepted for publication July 19, 1993.
$(20,21)$. However, no quantitative examination has yet been made of the changes in each major component of atherosclerotic lesions with progression of the disease, although quantitative analysis is needed to clarify the development of atherosclerosis.

This study investigated the changes of the major components of atherosclerotic lesions during progression from the early to advanced stage. We measured the areas of the major components (i.e., macrophages, smooth muscle cells, collagen fibers, and extracellular lipid deposits) found in atherosclerotic lesions in the aortas of WHHL rabbits, and examined the relationship between the changes in these components and the progression of atherosclerosis. 


\section{Materials and Methods}

\section{Animals}

Fourteen WHHL rabbits bred at Kobe University were used in this study. The animals were fed standard rabbit chow (Type CR-3, Clea Japan Inc., Tokyo) $120 \mathrm{~g} /$ day, and given water ad libitum. Four rabbits had early lesions (up to 3 months), five had transitional lesions (11-15 months), and five had advanced lesions (20-24 months). These animals had the average aortic surface lesional area for WHHL rabbits at each age (22). The rabbits were anesthetized with sodium pentobarbital and perfused with lactated Ringer's solution and Bouin's fixative at a constant pressure of $100 \mathrm{mmHg}$ using a perfusion apparatus (VPF-1, Nisshin EM, Tokyo). After perfusion fixation, the aortas were excised from the root of the ascending aorta down to the common iliac bifurcation. All experiments were conducted according to the "Guidelines for Animal Experiments Kobe University School of Medicine".

\section{Measurement of the lesional area}

The aortic surface lesional area was measured by the previously described method (23). Aortas were opened longitudinally along the anterior wall, the intimal surface area was copied, and the outlines of the intimal surface and the intimal lesions were traced on the photocopies. Then the intimal surface area and the lesional area were measured with a color image analyzer (SP 500, Olympus Co., Tokyo). The ratio of the lesional area to the intimal surface area was calculated for the aortic arch, the proximal and distal thoracic aorta, and the proximal and distal abdominal aorta.

\section{Histological examination}

The segments which showed the most severe lesions in each of the five parts of the aorta described above were embedded in paraffin and $4 \mu \mathrm{m}$ thick sections were prepared. Immunohistochemical staining with monoclonal antibody HHF35 (24) (a muscle actin-specific antibody) and monoclonal antibody RAM-11 (4) (a rabbit macrophage-specific antibody) was performed by the previously described method (4). The sections were also stained with hematoxylin and eosin, elastic van Gieson, Azan-Mallory, and Kossa stains. Sections were observed under a light microscope at a magnification of 4-20 times, and all lesional parameters (including the area of each lesional component) were measured using a color image analyzer. Cells featuring black reaction products after HHF35 staining were defined as smooth muscle cells, cells with black reaction products after RAM-11 staining were defined as macrophages, areas stained black with Kossa staining were defined as calcium deposits, and extracellular vacuoles or lacunae were defined as extracellular lipid deposits. In Azan-Mallory stained sections, fibers with three prime color elements (red, green and blue) in the color image analyzer were detected as elastin, while fibers having only a blue element were defined as collagen. The average intimal thickness was calculated by dividing the intimal lesional area by the length of the media (25). The percentage of each lesional component was calculated by dividing the area of each component by the intimal lesional area in each section of the aorta.

\section{Statistical analysis}

Data are reported as the mean \pm standard error. Statistical analysis was carried out using the Mann-Whitney utest for the percent lesional area, average intimal thickness, area of each lesional component, and percentage of each lesional component. The chi-square test was used to compare the incidence of cholesterol crystal deposition, calcification, and medial attenuation. Correlation coefficients between the average intimal thickness and the area of each lesional component or between the average intimal thickness and the percentage of each lesional component were assessed using Spearman rank correlation coefficients.

\section{Results}

Surface lesional area and intimal thickness of the aorta

Fig. 1 shows the surface lesional area of aortic atherosclerosis in WHHL rabbits. The most severe atherosclerotic lesions were observed in the aortic arch and the percent lesional area was $42 \pm 15 \%$ in young rabbits, $99 \pm$ $1 \%$ in middle-aged rabbits, and $100 \%$ in elderly rabbits. The severity of atherosclerosis decreased towards the distal portion of the aorta. The percent lesional area was significantly higher in middle-aged rabbits than in young rabbits for all parts of the aorta, except the distal abdominal aorta.

The average intimal thickness was also greatest in the aortic arch and was least in the distal abdominal aorta (Fig. 2). Intimal thickness increased significantly with age in the aortic arch, distal thoracic aorta, and proximal abdominal aorta $(p<0.05$ or $p<0.01$ ).

\section{Histological findings}

Fig. 3 shows the histological and immunohistochemical findings of early, transitional and advanced atherosclerotic lesions. In early lesions (Figs. $3 \mathrm{~A}-\mathrm{C}$ ), macrophages occupied almost the entire lesion and occasionally formed multiple layers of cells. In some cases, macrophages had already infiltrated the media and been transformed into foam cells even though there was no fragmentation of the internal elastic lamina. In such cases, smooth muscle cells around the foam cells in the media appeared to be oriented towards the intima. A few smooth muscle cells and some collagen fibers were observed at the base of the lesion and among the layers of macrophages. Extracellular lipid deposits were not observed.

In transitional lesions (Figs. 3D-F), numerous foam cells 


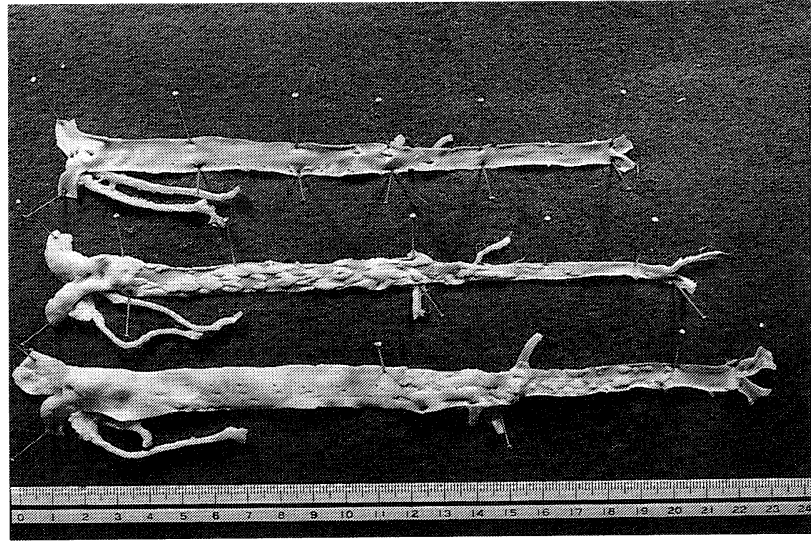

Fig. 1. Photographs of aortic atherosclerosis in WHHL rabbits, showing atherosclerotic lesions at the ages of 3,12 and 22 months from top to bottom, respectively.



Fig. 2. Thickness of the aortic intima. Intimal thickness was measured at the aortic arch $(\mathbf{\Delta})$, the proximal $(\bigcirc)$ and distal $(\bullet)$ portions of the thoracic aorta, and the proximal $(\square)$ and distal (a) portions of the abdominal aorta. Bars show the mean $\pm S$. E. Significant difference from the 3 month-old group $\left(p<0.05^{*}\right)$ and from the 11- to 15-month-old group $(p<0.05+$ and $p<0$. $01++$ ) by the Mann-Whitney U-test.

derived from macrophages were observed beneath the fibrous cap. Prominent foam cells were found to have accumulated in the media in the vicinity of the intimal lesions and smooth muscle cells were observed in the fibrous cap. In the media, the degree of staining with HHF35 (directed against smooth muscle cells) was reduced. Numerous collagen fibers were frequently observed in the upper and middle layers of the intimal lesions, while extracellular lipid deposits were observed in the middle and deeper layers.

Advanced lesions (Figs. 3G-I) featured the necrotic debris of foam cells, cholesteryl crystals, and calcification at sites deep in the intimal lesions. Newly infiltrating macrophages were observed above the fibrous cap. Collagen fibers were distributed throughout the lesions and numerous lipid accumulations were seen in the extracellular matrix.

\section{Quantitative analysis of each lesional component}

The average intimal thickness of the proximal thoracic aorta was $4.2 \pm 2.4 \mu \mathrm{m}$ in young rabbits, $228.2 \pm 31.2 \mu \mathrm{m}$ in middle-aged rabbits, and $430.8 \pm 88.0 \mu \mathrm{m}$ in elderly rabbits (Fig. 2). The aortic lesions at these ages were early, transitional and advanced, respectively. Accordingly, the proximal thoracic aorta was used for the following analyses.

Fig. 4 shows the changes in the area and percentage of each lesional component with the progression of atherosclerosis. The macrophage area increased significantly from $0.031 \pm 0.016 \mathrm{~mm}^{2}$ in early lesions to $0.762 \pm$ $0.118 \mathrm{~mm}^{2}$ in transitional lesions and $0.628 \pm 0.323 \mathrm{~mm}^{2}$ in advanced lesions. In contrast, the proportion of macrophage area decreased significantly from $62 \pm 3 \%$ in early lesions to $26 \pm 4 \%$ in transitional lesions and $10 \pm 4 \%$ in advanced lesions. The smooth muscle cell area also increased significantly from $0.001 \pm 0.001 \mathrm{~mm}^{2}$ in early lesion to $0.154 \pm 0.047 \mathrm{~mm}^{2}$ in transitional lesion and $0.100 \pm 0.011 \mathrm{~mm}^{2}$ in advanced lesions. However, the proportion of smooth muscle cell area changed very little, remaining between $2 \%$ and $4 \%$. The collagen fiber area increased significantly from $0.001 \pm 0.001 \mathrm{~mm}^{2}$ in early lesions to $0.360 \pm 0.059 \mathrm{~mm}^{2}$ in transitional lesions and $1.543 \pm 0.307 \mathrm{~mm}^{2}$ in advanced lesions, while the respective percentages were $1 \pm 1 \%, 13 \pm 3 \%$, and $26 \pm 4 \%$. The area of extracellular lipid deposits also increased significantly with the progression of lesions from early to advanced $\left(0 \mathrm{~mm}^{2}\right.$ vs. $\left.0.594 \pm 0.231 \mathrm{~mm}^{2}, \mathrm{p}<0.05\right)$, while the percentage increased from $0 \%$ to $8 \pm 2 \%(p<0.05)$. Calcification was observed in two rabbits with advanced lesions, but it comprised less than $3 \%$ of the lesional area. Similar results were obtained for the four other aortic regions (data not shown).

The average intimal thickness can be considered an index of the degree of progression of atherosclerosis. Table 1 shows the correlation coefficients between the average intimal thickness and each major lesional component. There were significant positive correlation coefficients between the thickness of intima and the collagen fiber area $(r=0.806, p<0.001)$, the area of extracellular lipid deposits $(r=0.862, p<0.001)$, the percentage of collagen fiber $(r=0.650, p<0.05)$, and the percentage of extracellular lipid deposits $(r=0.762, p<0.005)$. Although the macrophage area did not correlate with the average intimal thickness, the percent macrophage area was negatively correlated with the average intimal thick- 

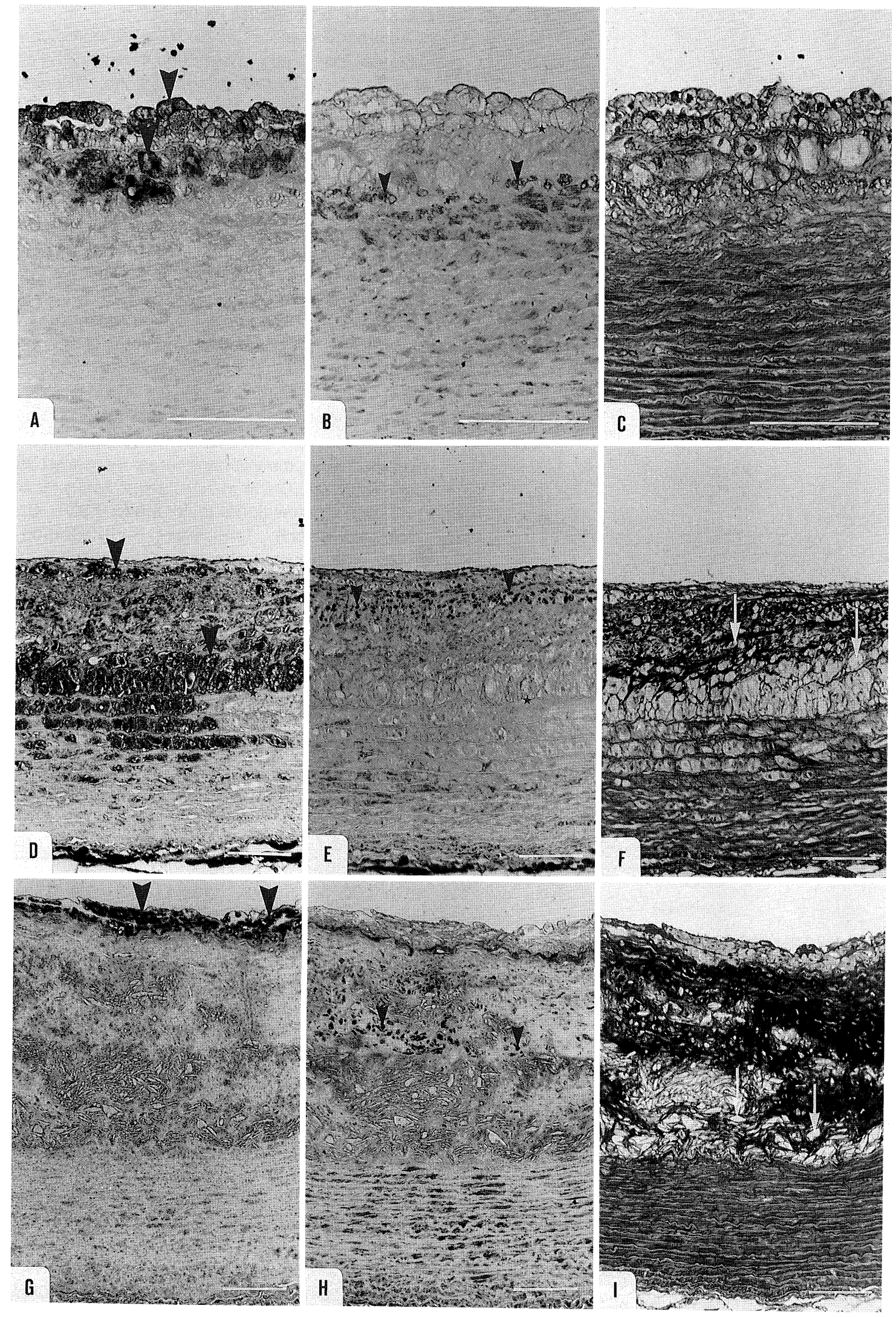
ness $(r=-0.846, p<0.001)$. Neither the smooth muscle cell area nor the percentage was correlated with the average intimal thickness. Similar results were also obtained in other portions of the aorta.

\section{Other histological findings}

Fragmentation and disappearance of the internal elastic lamina was observed in the advanced lesions of elderly rabbits, and these changes progressed as the lesions

(A)

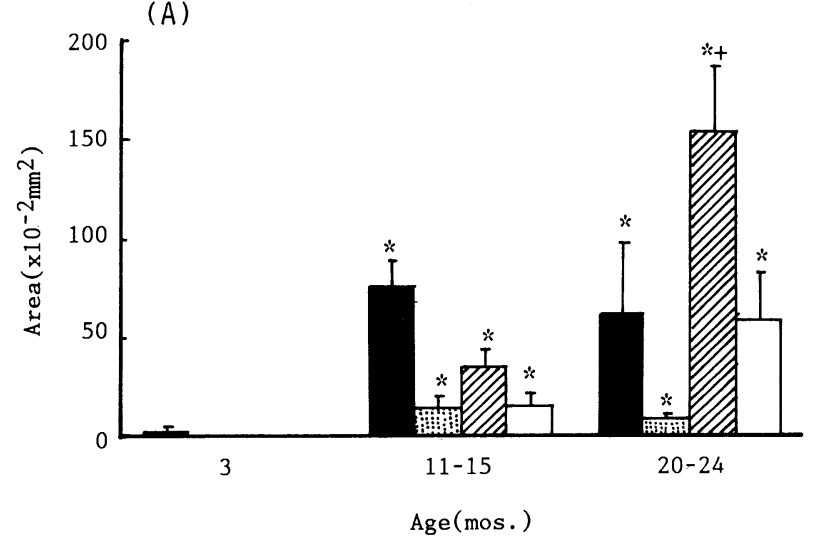

(B)

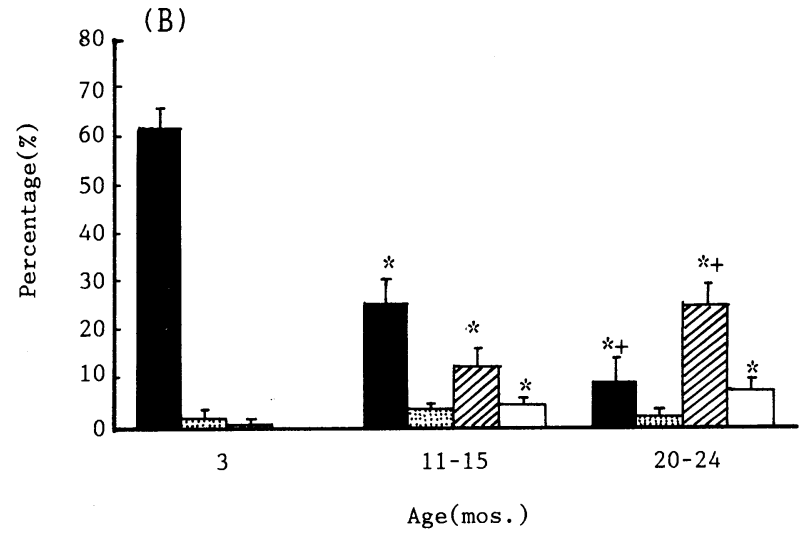

Fig. 4. Area of each component of the atherosclerotic lesions $(A)$ and the percent of lesional area $(B)$. Lesions in the proximal thoracic aorta were assessed. The percent of lesional area was calculated by dividing the area of each component by the intimal lesion area in each section of the aorta. घ, macrophages ; $\square$, smooth muscle cells ; $\square$, collagen fibers; $\square$, extracellular lipid deposits. Columns and bars represent the mean and standard error, respectively. Significant difference from the 3-month-old group $\left(p<0.05^{*}\right)$ and from the 11 - to 15 -month-old group $(p<0.05+)$ by the Mann-Whitney U-test. expanded. Deposition of cholesteryl crystals was observed in atheromatous plaques and in the fibrous layers deep within the intimal lesions. The incidence of cholesteryl crystal deposition was $80 \%$ in transitional lesions and $100 \%$ in advanced lesions. The incidence of medial attenuation was $60 \%$ in transitional lesions and $40 \%$ in advanced lesions. Mural thrombus was not observed in any of the sections.

\section{Discussion}

Histopathological and immunohistochemical studies of aortic atherosclerosis in WHHL rabbits were performed previously (3-7). However, there are no studies involving serial observation from the early fatty streak lesions to advanced complicated lesions, and no quantitative examination of changes in the major lesional components during the progression of atherosclerosis. In this study, we quantitatively analyzed the major components of the atherosclerotic lesion using a color image analyzer and examined the relationship of changes in these components to expansion of the atherosclerotic lesions.

In young $\mathrm{WHHL}$ rabbits, the atherosclerotic lesions in the aorta occurred at the orifices of the branching vessels and were $\mathrm{V}$-shaped, and the average intimal thickness was under $20 \mu \mathrm{m}$ (Figs. 1 and 2). Histological examination showed early fatty streaks (Figs. 3A-C), which corresponding with previous reports (3-5). In the early fatty streak lesions, macrophages were a major component (Fig. 4). Cybulsky and Gimbrone reported that adhesion molecules for mononuclear leukocytes are expressed by the aortic endothelium overlying early fatty streaks in WHHL and dietary hypercholesterolemic rabbits (26). Therefore, macrophages are considered to play an important role in the initiation of atherosclerosis. Some macrophages had infiltrated into the media and the smooth muscle cells around these macrophages were not stained by HHF35 (Figs. 3A, B). This finding was also observed in the transitional lesions (Figs. 3D, E). Smooth muscle cells in atherosclerotic lesions are heterogeneous in their specificity for various monoclonal antibodies (i.e., muscleactin-specific HHF35, smooth muscle-actin-specific CGA7 (27), and vimentin-specific $43 \beta \mathrm{H} 8(28)$ ). Ueda et al. reported that although normal smooth muscle cells were reactive with these three monoclonal antibodies, the reactivity of smooth muscle cells in fibrocellular tissue obtained after percutaneous transluminal coronary angioplasty showed considerable variability (29). Therefore, smooth muscle cells with a low reactivity to HHF35 may

Fig. 3. Photomicrographs of atherosclerotic lesions in the proximal thoracic aorta of WHHL rabbits. Macrophages (large arrowheads), smooth muscle cells (small arrowheads), extracellular lipid deposits (arrows), and internal elastic laminas (asterisks) can be seen. (A to C) Early lesions : (D to F) Transitional lesions: (G to I) Advanced lesions. Immunohistochemical staining with anti-macrophage monoclonal antibody RAM-11 (A, D and G), and with anti-smooth muscle cell monoclonal antibody HHF-35 (B, E and $\mathrm{H})$. Azan-Mallory staining (C, F and I). Bars $=100 \mu \mathrm{m}$. 
Table 1. Correlation coefficients between intimal thickness and the components of atherosclerosis in the proximal thoracic aorta of WHHL rabbits.

\begin{tabular}{lcccc}
\hline & Macrophages & $\begin{array}{c}\text { Smooth } \\
\text { muscle cells }\end{array}$ & $\begin{array}{c}\text { Collagen } \\
\text { fibers }\end{array}$ & $\begin{array}{c}\text { Extracellular } \\
\text { lipid deposits }\end{array}$ \\
\hline Lesional area of the component & 0.451 & 0.533 & 0.806 & 0.862 \\
Percent lesional area of the component & -0.846 & n.s. & $p<0.001$ & $p<0.001$ \\
& $\mathrm{p}<0.001$ & 0.122 & 0.650 & 0.762 \\
\hline
\end{tabular}

Correlation coefficients were calculated as Spearman rank correlation coefficients.

n.s., not significant ; $n=13$

have undergone a phenotypic conversion.

In middle-aged WHHL rabbits, atherosclerotic lesions extended from the aortic arch to the proximal abdominal aorta (Fig. 1) and the average intimal thickness was about $230 \mu \mathrm{m}$ in the proximal thoracic aorta (Fig. 2). Tsukada et al. reported that early fibrous plaques contain relatively equal numbers of smooth muscle cells and macrophages (4). In addition, Ross reported that macrophages, endothelium, and platelets secrete growth factors for smooth muscle cells which migrate from the media into the intima as a result of this stimulation(2). These studies suggest that both macrophages and smooth muscle cells play an important role in the progression of atherosclerosis from an early fatty streak lesion to a fibrous fatty streak. However, our study showed that the lesional area occupied by macrophages increased about fourfold when compared to the area occupied by smooth muscle cells (Figs. 3D-E and Fig. 4). This discrepancy may be caused by differences in the serum cholesterol level of WHHL rabbits from a different colony. The serum cholesterol levels of 12 -month-old WHHL rabbits was about $500 \mathrm{mg} / \mathrm{dl}$ in our colony and about $350 \mathrm{mg} / \mathrm{dl}$ in the Washington University colony (5). In any case, the role of macrophages in the progression of atherosclerosis from an early fatty streak to a transitional lesion appeared to be more prominent than that of smooth muscle cells in our WHHL rabbits.

In elderly WHHL rabbits, atherosclerotic lesions spread over the entire surface of the aorta (Fig. 1) and the average intimal thickness of the proximal thoracic aorta was about $430 \mu \mathrm{m} \quad$ (Fig. 2). Histological examination showed advanced complicated lesions (Figs. 3G-l). Although, the area occupied by macrophages and smooth muscle cells had not increased compared to that of transitional lesions, the area of collagen and extracellular lipid deposits increased about fourfold and threefold, respectively (Fig. 4). Distinguishing features at this stage were a marked increase in collagen and the disruption of foam cells. Progression of atherosclerosis from a transitional lesion to an advanced lesion is considered to occur as follows: 1) macrophages infiltrate the media and are subsequently transformed into foam cells (Fig. 3D), 2) these foam cells fracture the internal elastic lamina and are then disrupted themselves, and 3) atheroma is formed and fibrosis occurs (Figs. 3G-I).

Spagnoli et al. examined the effect of aging on atherogenesis (30). They compared atherosclerotic lesions induced by low-dose cholesterol feeding for 18 month in rabbits aged 4 months and rabbits aged 46 months, and found that young rabbits developed fatty streak lesions and older rabbits developed fibroatheromatous plaques despite having similar lipid levels. Therefore, the increase of fibrosis in the atherosclerotic lesions of WHHL rabbits over 20 months old may be related to aging. Serum cholesterol levels decreased from about $500 \mathrm{mg} / \mathrm{dl}$ at 12 months of age to about $350 \mathrm{mg} / \mathrm{dl}$ at over 20 months of age in our WHHL rabbits. A decrease of the serum cholesterol level with aging suggests that the amount of lipoprotein entering the aortic wall should also decrease, and this should suppress the proliferation of macrophages and smooth muscle cells in atherosclerotic lesions. These results suggest that both the disruption of foam cells and fibrosis may play an important role in the progression of atherosclerosis from a transitional lesion to an advanced lesion.

Aqel et al. (31) and Gown et al. (32) have reported immunohistochemical analyses of the cellular composition of human atherosclerosis. According to their studies, fibro-fatty lesions are composed almost exclusively of macrophages and have few smooth muscle cells, while advanced plaques are characterized by complex layers of macrophages and smooth muscle cells. Therefore, the cellular composition of aortic atherosclerosis in WHHL rabbits appears to be similar to that of human atherosclerosis.

In conclusion, this study showed that measurement of major lesional components using a color image analyzer is a feasible method of evaluating atherosclerotic lesions. This study also suggested that macrophages play an important role in atherogenesis and in the progression of lesions, and that the role of macrophages is more important than that of smooth muscle cells in the progression of aortic atherosclerosis. 
Acknowledgments: The authors are indebted to Mrs Megumi Tsutsumi-Okayama and Mr. Toshiaki Tamura, Ken-ichi Yoneda, Shinji Saruki and Seiji Katayama for their excellent technical assistance.

\section{References}

(1) Ross R and Glomset JA : The pathogenesis of atherosclerosis. N Engl J Med, 295 : 369-377, 1976

(2) Ross R: The pathogenesis of atherosclerosis - in update. N Engl J Med, 314: 488-500, 1986

(3) Buja LM, Kita T, Goldstein JL, Watanabe Y, and Brown MS : Cellular pathology of progressive atherosclerosis in the WHHL rabbit, an animal model of familial hypercholesterolemia. Arteriosclerosis, 3: 87-101, 1983

(4) Tsukada T, Rosenfeld ME, Ross R, and Gown AM: Immunocytochemical analysis of cellular components in atherosclerotic lesions: Use of monoclonal antibodies with the Watanabe and fat-fed rabbit. Arteriosclerosis, 6: 601-613, 1986

(5) Rosenfeld ME, Tsukada T, Gown AM, and Ross R: Fatty streak initiation in Watanabe heritable hyperlipidemic and comparably hypercholesterolemic fat-fed rabbits. Arteriosclerosis, 7 : 9-23, 1987

(6) Rosenfeld ME, Tsukada T, Chait A, Bierman EL, Gown $A M$, and Ross R: Fatty streak expansion and maturation in Watanabe heritable hyperlipidemic and comparably hypercholesterolemic fat-fed rabbits. Arteriosclerosis, 7: 24-34, 1987

(7) Rosenfeld ME and Ross R: Macrophage and smooth muscle cell proliferation in atherosclerotic lesions of WHHL and comparably hypercholesterolemic fat-fed rabbits. Arteriosclerosis, 10:680-687, 1990

(8) Watanabe $Y$ : Serial inbreeding of rabbits with hereditary hyperlipidemia (WHHL-rabbit): Incidence and development of atherosclerosis and xanthoma. Atherosclerosis, 36: 261-268, 1980

(9) Goldstein JL, Kita T, and Brown MS: Defective lipoprotein receptors and atherosclerosis: Lessons from an animal counterpart of familial hypercholesterolemia. $\mathrm{N}$ Engl J Med, 309: 288-296, 1983

(10) Davis HR, Vesselinovitch D, and Wissler RW: Histochemical detection and quantification of macrophages in rhesus and cynomolgus monkey atherosclerotic lesion. $\mathrm{J}$ Histochem Cytochem, 32 : 1319-1327, 1984

(11) Faggiotto A, Ross R, and Harker L: Studies of hypercholesterolemia in the nonhuman primate. 1. Changes that lead to fatty streak formation. Arteriosclerosis, 4 : 323-340, 1984

(12) Faggiotto A and Ross R: Studies of hypercholesterolemia in the nonhuman primate. II. Fatty streak conversion to fibrous plaque. Arteriosclerosis, 4:341-356, 1984

(13) Gerrity RG: The role of the monocyte in atherogenesis. I. Transition of blood-borne monocytes into foam cells in fatty lesions. Am J Pathol, 103: 181-190, 1981

(14) Gerrity RG: The role of the monocyte in atherogenesis. II. Migration of foam cells from atherosclerotic lesions. Am J Pathol, 103: 191-200, 1981

(15) Poole JCF and Florey HW: Changes in the endothelium of the aorta and the behavior of macrophages in experimental atheroma of rabbits. J Pathol Bacteriol, 75 :
$245-250,1958$

(16) Imai H, Lee KT, Patan S, Pamilio E, Florentin R, and Thomas WA: Atherosclerosis in rabbits: architectural and subcellular alterations of smooth muscle cells of aortas in response to hyperlipidemia. Exp Mol Pathol, 5: 273-310, 1966

(17) Gaton E and Wolman M: The role of smooth muscle cells and hematogenous macrophages in atheroma. $\mathrm{J}$ Pathol, 123: 123-128, 1977

(18) Watanabe T, Hirata M, Yoshikawa $Y$, Nagafuchi $Y$, Toyoshima $\mathrm{H}$, and Watanabe $\mathrm{T}$ : Role of macrophages in atherosclerosis: sequential observation of cholesterolinduced rabbit aortic lesion by the immunoperoxidase technique using monoclonal antimacrophage antibody. Lab Invest, 52: 80-90, 1985

(19) Joris I, Zand T, Nunnari JJ, Krolikowski FJ, and Malno G : Studies on the pathogenesis of atherosclerosis. I. Adhesion and emigration of mononuclear cells in the aorta of hypercholesterolemic rats. Am J Pathol, 113: 341-358, 1983

(20) Jerome WG and Lewis JC: Early atherogenesis in white Carneau pigeons. I. Leukocyte margination and endothelial alterations at the celiac bifurcation. Am J Pathol, 116 : 56-68, 1984

(21) Jerome WG and Lewis JC: Early atherogenesis in white Careau pigeons. II. Ultrastructural and cytochemical observations. Am J Pathol, 119: 210-222, 1985

(22) Shiomi $M$, Ito $T$, Shiraishi $M$, and Watanabe $Y$ : Inheritability of atherosclerosis and the role of lipoproteins as risk factors in the development of atherosclerosis in WHHL rabbits : Risk factors related to coronary atherosclerosis are different from those related to aortic atherosclerosis. Atherosclerosis, 96 : 43-52, 1992

(23) Watanabe $Y$, Ito $T$, Shiomi M, Tsujita $Y$, Kuroda $M$, Arai $M$, Fukami $M$, and Tamura $A$ : Preventive effect of pravastatin sodium, a potent inhibitor of 3-hydroxy-3methylglutaryl coenzyme $A$ reductase, on coronary atherosclerosis and xanthoma in WHHL rabbits. Biochim Biophys Acta, 960 : 294-302, 1988

(24) Tsukada T, Tippenes D, Mar H, Gordon D, Ross R, and Gown AM: HHF35: a muscle-actin-specific monoclonal antibody. Am J Pathol, 126: 51-60, 1987

(25) Shiomi M, Ito T, Watanabe $Y$, Tsujita $Y$, Kuroda M, Arai M, Fukami M, Fukushige J, and Tamura A : Suppression of established atherosclerosis and xanthomas in mature WHHL rabbits by keeping their serum cholesterol levels extremely low. Atherosclerosis, 83: 69-80, 1990

(26) Cybulsky ML and Gimbrone MA : Endothelial expression of a mononuclear leukocyte adhesion molecule during atherogenesis. Science, 251: 788-791, 1991

(27) Gown AM, Vogel AM, Gordon D, and Lu PL: A smooth muscle-specific monoclonal antibody recognizes muscle-specific actin isozymes. J Cell Biol, 100 : 807813, 1985

(28) Gown AM and Vogel AM: Monoclonal antibodies to intermediate filament proteins of human cells: Unique and cross-reacting antibodies. J Cell Biol, 95: 414424, 1982

(29) Ueda M, Becker AE, Tsukada T, Numano F, and Fujimoto $T$ : Fibrocellular tissue response after percutaneous transluminal coronary angioplasty, an immunocytochemical analysis of the cellular composition. Circulation, 83: 1327-1332, 1991

(30) Spagnoli LG, Orlandi A, Mauriello A, Santeusanio G, De 
Angelis C, Lucreziotti R, and Ramacci MT: Aging and atherosclerosis in the rabbit: 1 . Distribution, prevalence and morphology of atherosclerotic lesions. Atherosclerosis, 89: 11-24, 1991

(31) Aqel NM, Ball RY, Waldmann $\mathrm{H}$, and Mitchinson $\mathrm{MJ}$ : Identification of macrophages and smooth muscle cells in human atherosclerosis using monoclonal antibodies. J Pathol, 146: 197-204, 1985

(32) Gown AM, Tsukada T, and Ross R: Human atherosclerosis. II. Immunocytochemical analysis of the cellular composition of human atherosclerotic lesions. Am J Pathol, 125 : 191-207, 1986 